\title{
Lymphokine-activated killer cell transplantation after anti-cancer treatment in two aged cats
}

\author{
Noritaka Maeta $^{1,2^{* \dagger}}$, Katsutoshi Tamura ${ }^{1 \dagger}$, Hiroshi Takemitsu ${ }^{1}$ and Masahiro Miyabe ${ }^{1,2}$ \\ 1Aikouishida Animal Hospital, Isehara, Kanagawa, Japan \\ ${ }^{2}$ Faculty of Veterinary Medicine, Okayama University of Science, Imabari, Ehime, Japan \\ ${ }^{\dagger}$ These authors contributed equally to this work.
}

\begin{abstract}
Immunotherapy improves both survival and remission rates after cancer surgery in humans, but its veterinary use has been limited. We determined the safety and feasibility of lymphokine-activated killer (LAK) cell transplantation in two aged cats that had undergone surgery for malignancy. Case 1 involved an 18-year-old male Japanese domestic cat. The cat exhibited appetite loss and poor physical activity after the surgical excision of oral squamous cell carcinoma followed by four sessions of radiotherapy, and the owner strongly requested immunotherapy for preventing further deterioration in the animal's quality of life (QOL). We subsequently administered LAK cells three times during a 2-month period. Case 2 involved a 20 -year-old female Japanese domestic cat who had undergone mammectomy after a diagnosis of breast adenocarcinoma. The owner strongly requested immunotherapy for QOL maintenance. We administered LAK cells four times over a period of 5 months. Autologous peripheral blood mononuclear cells (PBMCs) fractionated using density gradient centrifugation were cultured in the media containing a high concentration of interleukin-2 and supplemented with $2.5 \%$ fetal calf serum. The derived LAK cells were centrifuged, suspended in $10 \mathrm{ml}$ of saline containing $1 \%$ of the subject's own blood, and infused into the cephalic vein of the cats over 30 min. The composition ratios of CD3, CD4, CD8, and CD21 were evaluated by flow cytometry. Bacterial culture and endotoxin testing for a sample of LAK cells showed negative results in both the cases. The leukocyte and erythrocyte counts and the body temperature were assessed on days 7, 14, and 21 after the transfusion. No abnormal signs were observed in either case, which confirmed the safety of the procedure. QOL scores showed no significant changes after the treatment, and the body temperature remained steady throughout the treatment. The findings from these cases suggest that the transplantation of LAK cells derived from PBMCs may be safe and feasible for use in cats, regardless of their age.
\end{abstract}

Keywords: CD4-CD8 ratio, Immunotherapy, Lymphokine-activated killer cells, T lymphocytes, Transplantation.

\section{Introduction}

Surgical intervention, radiotherapy, and chemotherapy are the mainstream cancer treatments for most animals. However, immunotherapy as a novel cancer treatment modality has recently received increased interest and has been implemented in clinical settings following investigations of the biological properties of human cell lines. Several studies on immunotherapy for humans have shown significant improvements in survival and remission rates over 2-5 years (Kimura et al., 2008; Hong et al., 2010). However, similar studies in veterinary medicine have been limited (O'Connor et al., 2012). Furthermore, no study has investigated the effects of immunotherapy in aged animals, who exhibit a much higher risk of tumor development and recurrence.

In human medicine, lymphokine-activated killer (LAK) cell therapy is administered for improvement of the immune response and prevention of recurrence and metastasis after the cancer resection (Mie et al., 2016).
In veterinary medicine, it was reported that the LAK cell transplantation in healthy dogs was safe and improved the immune response (Hoshino et al., 2008). Among LAK cells derived from the cats, prominent cytotoxicity of $\mathrm{CD}^{+}$and $\mathrm{CD} 57^{+}$cells was observed in an in vitro study by Zhao et al. (1995).

However, there are no in vivo reports on the efficacy and safety of LAK cell transplantation in cats. Accordingly, we aimed to determine the safety and feasibility of LAK cell transplantation used as supportive therapy in two aged cats with a history of treatment for malignancy.

\section{Case Details}

The procedures were conducted in accordance with the Japanese Regulations for Animal Welfare issued by The Ministry of Education, Culture, Sports, Sciences, and Technology of Japan. Both owners provided informed consent for all the procedures before these were undertaken. No animals are identifiable in this publication, so additional informed consent for publication was not required. 
Case 1 involved an 18-year-old male Japanese domestic cat who had undergone surgical excision of oral squamous cell carcinoma followed by four sessions of radiotherapy. The treatment had resulted in loss of appetite and lethargy, so the owner strongly requested immunotherapy to prevent further deterioration in the animal's quality of life (QOL). No obvious metastasis was observed at this time. Subsequently, we transfused LAK cells three times during a 2-month period. Case 2 involved a 20-year-old female Japanese domestic cat who had undergone mammectomy for malignant breast adenocarcinoma. The owner strongly requested immunotherapy to prevent QOL deterioration. No obvious metastasis was observed at this time. Subsequently, we transfused LAK cells four times over a period of 5 months.

The LAK cells were prepared for transfusion as follows: Peripheral blood mononuclear cells (PBMCs) were isolated by density gradient centrifugation of 5 $\mathrm{ml}$ of blood collected from the cephalic vein of each patient. The sample was prepared with the addition of 5 $\mathrm{ml}$ of RPMI1640 culture medium (Cosmo Bio Co. Ltd., Tokyo, Japan) and $5 \mathrm{ml}$ of Lymphoprep ${ }^{\mathrm{TM}}$ (density 1.077; Nycomed Pharma Pvt Ltd, Mumbai, India).

The isolated layers of mononuclear cells were transferred onto $5 \mathrm{ml}$ of RAM1 medium (Canine-lab Corporation) for culture at $37^{\circ} \mathrm{C}$ in $5 \%$ carbon dioxide. On the second, fourth, and sixth days of culture, $5 \mathrm{ml}$ of RAM2 solution (Canine-lab Corporation) was added. The total cultivation time was 10 and 14 days. The resulting LAK cells were rinsed with $15 \mathrm{ml}$ RPMI1640 medium and subjected to centrifugation for $10 \mathrm{~min}$ at $1,600 \mathrm{rpm}$. This was repeated twice before its suspension in $10 \mathrm{ml}$ of saline containing $1 \%$ of the subject's own blood for transfusion via the cephalic vein over $30 \mathrm{~min}$.

A sample of the resulting solution was subjected to flow cytometry using the antibodies listed in Table 1 for evaluation of the antigenic phenotypes of the cells. We added $20 \mu \mathrm{l}$ of phycoerythrin (PE)-conjugated CD8 and CD21 or fluorescein isothiocyanate (FITC)-conjugated CD4 and CD3 to $100 \mu 1$ of saline solution suspended with LAK cells $\left(1 \times 10^{5}\right.$ cells $)$. These samples were incubated for $30 \mathrm{~min}$ in the dark and subsequently mixed thoroughly with $900 \mu \mathrm{l}$ of phosphate-buffered saline. The sample was evaluated over 100,000 events. The results of flow cytometry for cases 1 and 2 are as shown in Table 2. Relative ratios (\%) were calculated for $\mathrm{CD}^{+}$lymphocytes ( $\mathrm{T}$ cells), $\mathrm{CD} 4^{+}$lymphocytes (helper T cells), $\mathrm{CD}^{+}$lymphocytes (cytotoxic $\mathrm{T}$ cells), and $\mathrm{CD} 21^{+}$lymphocytes (B cells), and the mean values for the ratios after each culture were derived. In both cases, $\mathrm{CD}^{+}$lymphocytes and, particularly, $\mathrm{CD} 4^{+}$ lymphocytes exhibited an increase, which resulted in an elevated $\mathrm{CD}^{+} / \mathrm{CD}^{+}$ratio.

A portion of each prepared sample was also subjected to endotoxin testing and bacterial culture. For the latter, LAK cell media were inoculated onto trypto- soya agar (SCD agar, Nissui Pharmaceutical Co., Ltd, Tokyo, Japan) and incubated at $30^{\circ} \mathrm{C}$ for 7 days. Endotoxin testing was performed in accordance with the method described by Tamura et al. (2012). Briefly, a colorimetric assay was performed using Toxicolor (Seikagaku Corporation) to confirm the absence of pyrogens (endotoxins) before transfusion. The results of both bacterial culture and endotoxin testing were negative.

The owners were instructed to monitor QOL of their respective pets before, and 3 and 7 days after the transfusion. The assessed parameters included body temperature, activity, appetite, vomiting, and defecation. The owners were asked to perform activity assessments twice a week and assign scores using a five-point scale as follows: 1 , none; 2 , rare; 3 , normal; 4, more than normal, and 5, high. Appetite was scored as: 1 , lack of appetite; 2 , slight loss; 3 , normal; 4 , more than normal, and 5, large appetite. Vomiting was scored as: 1,10 or more times a day; 2 , six or more times a day; 3 , two or more times a day; 4 , once a day and 5 , no vomiting. Defecation was scored as follows: 1 , diarrhea occurring multiple times; 2 , diarrhea occurring once a day; 3 , soft stool observed multiple times a day; 4, soft stool observed once a day, and 5, normal stool. Comparisons of scores before and after the treatment showed no significant differences, and the body temperature remained steady throughout the course of the therapy for both cats.

In addition, physical examinations were performed and leucocyte and erythrocyte counts were measured along with the body temperature on days 7, 14, and 21 after the transfusion. No abnormal signs were observed in either case, which confirmed the safety of the procedure.

\section{Discussion}

Immunological surveillance and the specific role of the lymphocytic immune system in the monitoring and elimination of tumor cells were first described by Burnet (1970). LAK cell therapy has been applied in the field of human medicine since the late 1980s; however, to the best of our knowledge, there are no studies of its application in cats. Accordingly, the present case report is the first to document the safety and feasibility of LAK cell transplantation in elderly cats.

LAK cell therapy is a technique whereby the lymphocyte count is increased in an in vitro environment with interleukin (IL)-2, and it has become the basis of numerous immunotherapy assays that are currently in use.

Ohno et al. (1992) reported that the $\mathrm{CD} 4^{+} / \mathrm{CD}^{+}$ratio in feline peripheral blood is $2.03 \pm 0.46$. In our study, the $\mathrm{CD}^{+} / \mathrm{CD}^{+}$ratios for cases 1 and 2 were $0.49 \pm 0.15$ and $0.82 \pm 0.17$, respectively. These comparatively low values can be attributed to the old age of the cats and the presence of underlying disease.

In both cases, phenotypic analysis showed an increase in $\mathrm{CD}^{+}\left(\mathrm{CD} 8^{-}\right)$lymphocytes and the $\mathrm{CD} 4^{+} / \mathrm{CD} 8^{+}$ratio 
Table 1. Monoclonal antibodies used in flow cytometry for evaluation of the antigenic phenotypes of cells.

\begin{tabular}{lllllll}
\hline Phenotype & Specificity & Host & Isotype & Conjugate & Clone & Manufacturer \\
\hline CD21 & B cells & Mouse & IgG1 & RPE $^{*}$ & CA2.1D6 & Serotec $^{\ddagger}$ \\
CD3 & T cells & Mouse & IgG1 & FITC & 5G-6-7-3 & CLC $^{\ddagger}$ \\
CD4 & Helper T cells & Mouse & IgG2a & FITC & Vpg34 & Serotec $^{\ddagger}$ \\
CD8 & Cytotoxic T cells & Mouse & IgG1 & RPE & Vpg9 & Serotec $^{\S}$ \\
\hline
\end{tabular}

*Rrhodophyceae phycoerythrin; †fluorescein isothiocyanate isomer I; †Serotec; §Canine-lab Corporation.

Table 2. Phenotypes of T lymphocytes for two aged cats that received LAK cell transplantation.

\begin{tabular}{lcccc}
\hline Phenotype (\%) & Case 1 Pre $^{*}$ & Case 1 Post $^{\dagger}$ & Case 2 Pre $^{*}$ & Case 2 Post $^{\dagger}$ \\
\hline $\mathrm{CD}^{+}$lymphocytes & $60.2 \pm 16.8$ & $71.2 \pm 23.5$ & $56.1 \pm 31.0$ & $74.7 \pm 11.9$ \\
$\mathrm{CD}^{+} 1^{+}\left(\mathrm{CD} 3^{-}\right)$lymphocytes & $15.9 \pm 9.8$ & $14.0 \pm 13.2$ & $14.4 \pm 5.5$ & $7.6 \pm 4.1$ \\
$\mathrm{CD}^{+}\left(\mathrm{CD} 8^{-}\right)$lymphocytes & $17.6 \pm 8.1$ & $33.1 \pm 19.9$ & $23.7 \pm 14.9$ & $47.3 \pm 9.7$ \\
$\mathrm{CD}^{+}\left(\mathrm{CD} 4^{-}\right)$lymphocytes & $35.2 \pm 10.6$ & $31.0 \pm 23.2$ & $27.1 \pm 14.7$ & $17.2 \pm 9.8$ \\
$\mathrm{CD}^{+} / \mathrm{CD}^{+}$ratio & $0.49 \pm 0.15$ & $2.17 \pm 2.81$ & $0.82 \pm 0.17$ & $4.47 \pm 4.56$ \\
\hline
\end{tabular}

All values are expressed as means \pm standard deviations; *peripheral blood mononuclear cells; †lymphokine-activated killer cells.

achieved by PMBC stimulation in the IL-2 containing medium, thus confirming the production of LAK cells from PBMCs.

In dog models, LAK cells were found to exhibit considerable cytotoxicity against tumor cells in vitro (Helfand et al., 1994), and this was largely attributed to the presence of $\mathrm{CD}^{+}$antibodies (Lin et al., 2010). Similar cytotoxicity of cat-derived $\mathrm{CD}^{+}$cells in vitro has been reported (Zhao et al., 1995) although no assays for evaluation of the in vivo cytotoxicity have been attempted in cat models.

IL-4 has an antiapoptotic effect and can suppress the production of tumor necrosis factor alpha (Racke et al., 1991). $\mathrm{CD}^{+}$cells can produce IL-4, and the analysis of our cases revealed that $33.1 \% \pm 19.9 \%$ and $47.3 \%$ $\pm 9.7 \%$ of the samples for cases 1 and 2 , respectively, comprised $\mathrm{CD}^{+}$cells. These high-cell proportions probably played a role in the prevention of QOL deterioration.

We attempted LAK cell transplantation in the two elderly cats with the aim of establishing a standardized immunotherapy regimen for cancer in cats. QOL of both cats was sustained throughout the course of treatment. Moreover, no side effects were observed in either case. Generally, QOL worsens in older animals receiving treatment for cancer; thus, measures for maintaining QOL are very important in such cases. Our findings suggest that LAK cell therapy may be useful for maintaining QOL during or after the cancer treatment. Future research should not only investigate the anticancer activity of LAK cells but also perform objective assessments of the treatment efficacy in cats.

\section{Conclusions}

In conclusion, we presented the first report on the safety and feasibility of LAK cell transplantation in aged cats.
The findings suggest that the transplantation of LAK cells derived from PBMCs may be safe and feasible for postoperative QOL maintenance in cats, regardless of their age.

\section{Conflict of interest}

The authors declare that there is no conflict of interest.

\section{References}

Burnet, F.M. 1970. The concept of immunological surveillance. Prog. Exp. Tumor. Res. 13, 1-27.

Helfand, S.C., Soergel, S.A., Modiano, J.F., Hank, J.A. and Sondel, P.M. 1994. Induction of lymphokineactivated killer (LAK) activity in canine lymphocytes with low dose human recombinant interleukin-2 in vitro. Cancer Biother. 9, 237-244.

Hong, J.J., Rosenberg, S.A., Dudley, M.E., Yang, J.C., White, D.E., Butman, J.A. and Sherry, R.M. 2010. Successful treatment of melanoma brain metastases with adoptive cell therapy. Clin. Cancer Res. 16, 4892-4898.

Hoshino, Y., Takagi, S., Osaki, T., Okumura, M. and Fujinaga, T. 2008. Phenotypic analysis and effects of sequential administration of activated canine lymphocytes on healthy beagles. J. Vet. Med. S. 70, 581-588.

Kimura, H., Iizasa, T., Ishikawa, A., Shingyouji, M., Yoshino, M., Kimura, M., Inada, Y. and Matsabayashi, K. 2008. Prospective phase II study of post-surgical adjuvant chemo-immunotherapy using autologous dendritic cells and activated killer cells from tissue culture of tumor-draining lymph nodes in primary lung cancer patients. Anticancer Res. 28, 1229-1238.

Lin, Y.C., Huang, Y.C., Wang, Y.S., Juang, R.H., Liao, K.-W. and Chu, R.-M. 2010. Canine CD8 T cells showing NK cytotoxic activity express mRNAs 
for NK cell-associated surface molecules. Vet. Immunol. Immunopathol. 133, 144-153.

Mie, K., Shimada, T., Akiyoshi, H., Hayashi, A. and Ohashi, F. 2016. Change in peripheral blood lymphocyte count in dogs following adoptive immunotherapy using lymphokine-activated $\mathrm{T}$ killer cells combined with palliative tumor section. Vet. Immunol. Immunopathol. 117, 58-63.

O’Connor, C.M., Sheppard, S., Hartline, C.A., Huls, H., Johnson, M., Palla, S.L., Maiti, S., Ma, W., Davis, R.E., Craig, S., Lee, D.A., Champlin, R., Wilson, H. and Cooper, L.J.N. 2012. Adoptive T-cell therapy improves treatment of canine nonHodgkin lymphoma post chemotherapy. Sci. Rep. 2, 249 .

Ohno, K., Watari, T., Goitsuka, R., Tsujimoto, H. and Hasegawa, A. 1992. Altered surface antigen expression on peripheral blood mononuclear cells in cats infected with feline immunodeficiency virus. J. Vet. Med. Sci. 54, 517-522.

Racke, M.K., Bonomo, A., Scott, D.E., Cannella, B., Levine, A., Raine, C.S., Shevach, E.M. and Röcken, M. 1991. Cytokine-induced immune deviation as a therapy for inflammatory autoimmune disease. J. Exp. Med. 180, 1961-1966.

Tamura, K., Harada, Y., Nagashima, N., Itoi, T., Ishino, H., Yogo, T., Nezu, Y., Hara, Y., Suzuki, Y., Ide, C. and Tagawa, M. 2012. Autotransplanting of bone marrow-derived mononuclear cells for complete cases of canine paraplegia and loss of pain perception, secondary to intervertebral disc herniation. Exp. Clin. Transplant. 10, 263-272.

Zhao, Y., Gebhard, D., English, R., Sellon, R., Tompkins, M. and Tompkins, W. 1995. Enhanced expression of novel $\mathrm{CD} 57^{+} \mathrm{CD} 8^{+} \mathrm{LAK}$ cells from cats infected with feline immunodeficiency virus. J. Leukoc. Biol. 58, 423-431. 